\title{
Is the French palliative care policy effective everywhere? Geographic variation in changes in inpatient death rates among older patients in France, 2010-2013
}

\author{
William B. Weeks ${ }^{1,2,3}$, Bruno Ventelou ${ }^{3}$, Marc Karim Bendiane $^{4,5,6}$ \\ ${ }^{1}$ The Dartmouth Institute for Health Policy and Clinical Practice, Lebanon, New Hampshire, USA; ${ }^{2}$ The Geisel School of Medicine, Hanover, New \\ Hampshire, USA; ${ }^{3}$ Aix-Marseille University (Aix-Marseille School of Economics), CNRS \& EHESS, Marseille, France; ${ }^{4}$ INSERM, UMR_S 912, \\ « Sciences Economiques \& Sociales de la Santé et Traitement de l'Information Médicale » (SESSTIM), F-13385, Marseille, France; ${ }^{5}$ Aix Marseille \\ Université, UMR_S 912, IRD, Marseille, F-13385, Marseille, France; ${ }^{6}$ ORS PACA, Observatoire Régional de la Santé Provence-Alpes-Côte d'Azur, \\ F-13006, Marseille, France \\ Contributions: (I) Conception and design: All authors; (II) Administrative support: None; (III) Provision of study materials or patients: B Ventelou; \\ (IV) Collection and assembly of data: WB Weeks; (V) Data analysis and interpretation: All authors; (VI) Manuscript writing: All authors; (VII) Final \\ approval of manuscript: All authors. \\ Correspondence to: Dr. William B. Weeks. The Dartmouth Institute for Health Policy and Clinical Practice, Lebanon, New Hampshire, USA. \\ Email: wbw@dartmouth.edu.
}

Background: Recently, French policymakers have tried to improve care at the end-of-life, by improving access to community-based palliative care, particularly for patients with cancer and neurological diseases. If effective, these efforts should reduce the proportion of such patients who die in the hospital. In light of these policies, we sought to determine the effectiveness of these efforts on reducing inpatient deaths by conducting a retrospective, observational analysis of patients aged 65 and older who were admitted to hospitals in France between 2010 and 2013 for 1 of 3 non-surgical conditions.

Methods: We calculated department-specific age- and sex-adjusted inpatient death rates for 3 types of nonsurgical admissions and modeled expected number of inpatient deaths had their rates for patients with cancer or neurological disease tracked those of patients with non-cancer non-neurological diseases.

Results: We found that patients admitted with a cancer diagnosis experienced 20,394 (13.0\%) fewer inpatient deaths that expected had non-surgical cancer diagnosis admission rates tracked those of nonsurgical non-cancer and non-neurological admission rates; patients admitted with a primary neurological disease diagnosis experienced 513 (4.5\%) fewer inpatient deaths than expected. During the study period, observed-to-expected inpatient deaths fell more dramatically and consistently for patients admitted with cancer diagnoses than for those admitted with neurological diseases. Observed-to-expected ratios fell least in departments that were on the periphery of the French mainland.

Conclusions: Our findings suggest that, in France, efforts to reduce inpatient death rates among patients with cancer or neurological disease diagnoses appear to be effective. However, their effectiveness varies geographically, suggesting that targeted efforts to improve lower performing departments may generate substantial performance improvements.

Keywords: Palliative care; cancer; France; health policy

Submitted Jun 21, 2016. Accepted for publication Jul 07, 2016.

doi: 10.21037/apm.2016.08.05

View this article at: http://dx.doi.org/10.21037/apm.2016.08.05 


\section{Introduction}

The World Health Organization has identified improving care at the end-of-life as a global health priority, with improved access to palliative care as a mechanism for doing so (1). High inpatient death rates may reflect overuse of healthcare resources among patients for whom endof-life care might be improved; and, in the mid-2000s, France has a relatively high inpatient death rate (2). Over the past decade, French policymakers have taken steps to improve care at the end-of-life. Actions have included passing a "Patient's Rights and End-of-life Care" Act in 2005 that clarified end-of-life medical practice in France by authorizing withholding or withdrawal of treatments when appropriate (3), implementing a national 4-year plan to develop home care for end-of life patients in 2008 (4), establishing the "Observatoire National de la Fin de Vie" in 2010 (5), and taking steps to encourage patients with cancer and neurological diseases to die at home, instead of in the hospital, including changing reimbursement to discourage the use of inpatient palliative care beds, transitioning palliative care efforts to the outpatient setting, and establishing palliative care networks that coordinate home care for these patients (6).

We wondered whether these efforts to improve access to palliative care were effective at reducing the proportion of patients who die in the hospital. We reasoned that, if they were, inpatient death rates for older patients admitted with cancer or neurological diseases would decline, over time, relative to those for patients admitted for other reasons. Therefore, we examined a retrospective observation study of the rates of inpatient deaths for patients admitted with cancer diagnoses, neurological disease diagnoses, and noncancer non-neurological disease diagnoses between 2010 and 2013.

\section{Methods}

From the Agence Technique de l'Information sur l'Hospitalisation, we obtained individual case-level data on all medical, surgical, and obstetrical discharges from all hospitals in mainland France for 2009 through 2013. Those data include the patient's age, the primary ICD-10 admission diagnosis, the groupes homogènes de malades (GHM) code (a diagnosis related group-like code), and the mode of discharge (including death).

We sought to examine the impact of these policy changes on patients aged 65 and older, because they had a disproportionately high number of inpatient deaths $(1,034,820$ of $1,363,820$, or $75.9 \%)$ relative to the incidence of their admissions for cancer $(11,043,400$ of $23,263,126$, or $47.5 \%)$, neurological diseases $(1,249,852$ of $3,814,873$, or $32.8 \%$ ), or non-cancer non-neurological diseases $(30,761,044$ of $85,239,965$, or $36.1 \%)$ during the study period. Reasoning that patients who were admitted for surgical interventions were not near the end-of-life, we further limited our analysis to patients who were not admitted for a surgical reason [those patients whose GHM code did not include the letter " $C$ " in the third position (7), representing $34,308,609(79.7 \%)$ of the $43,054,296$ admissions among patients aged 65 and older between 2010 and 2013].

Because demographics vary geographically in France (8-10), Dartmouth Atlas Project (11) methods to calculate age- and sex-adjusted admission rates for 96 geographicallydefined departments in mainland France. For each year, we obtained age- and sex-specific department-level population estimates from the French census (12). We analyzed three categories of admission: cancer admissions [non-surgical admission with a primary diagnosis of cancer (ICD10 diagnosis beginning with "C")]; neurological disease admissions [non-surgical admission with a primary diagnosis of a neurological disorder (ICD-10 diagnosis beginning with "G")]; and non-cancer non-neurological disease admissions (non-surgical admissions without a primary diagnosis of cancer or a neurological disease). We identified inpatient deaths within each type of admission from the MODE_SORTEE variable (where "9" indicates inpatient death).

The age- and sex-adjusted rates of admission for the 3 different categories we examined changed over the study time period (Table 1). To account for secular changes, we used year- and department-specific age- and sex-adjusted inpatient death rates for non-cancer non-neurological disease admissions to model the expected number of inpatient admissions for patients with cancer or neurological diseases, had they changed at the same rate as non-cancer non-neurological disease admissions did. We then applied inpatient death rates for cancer and neurological diseaserelated admissions to the expected number of admissions to calculate the expected number of age- and sex-adjusted inpatient deaths for these admission types. Finally, so that we could identify geographic differences, we determined whether and how much the observed number of age- and sex-adjusted inpatient deaths differed from the expected number, at the department level. The process that we used 
Table 1 French national age- and sex-adjusted rates (per 1,000 patients) of non-surgical admissions for patients admitted with a primary diagnosis of cancer, neurological disease, or a non-cancer non-neurological disease among patients aged 65 and older in 2010-2013, and comparison of 2013 to 2010 rates

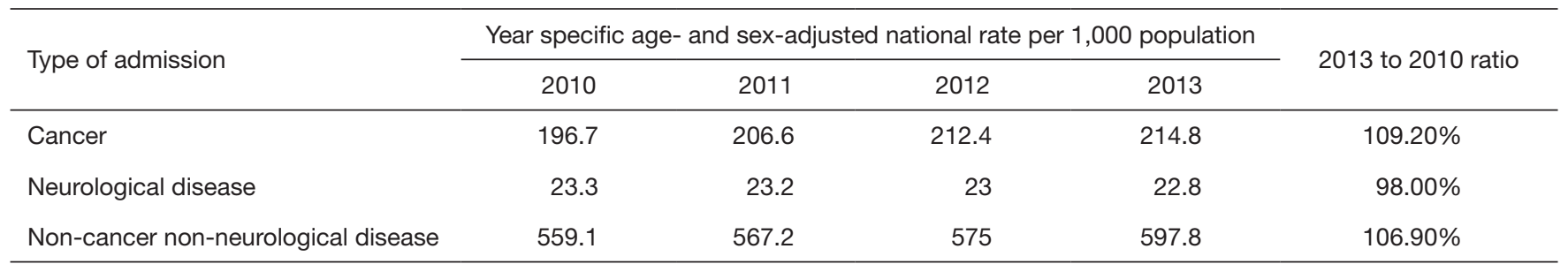

Table 2 Method for creating an expected rate of cancer admissions and deaths for comparison to non-cancer non-neurological disease admissions and deaths among patients aged 65 and older at the department level with an example given for Ain (department number 01)

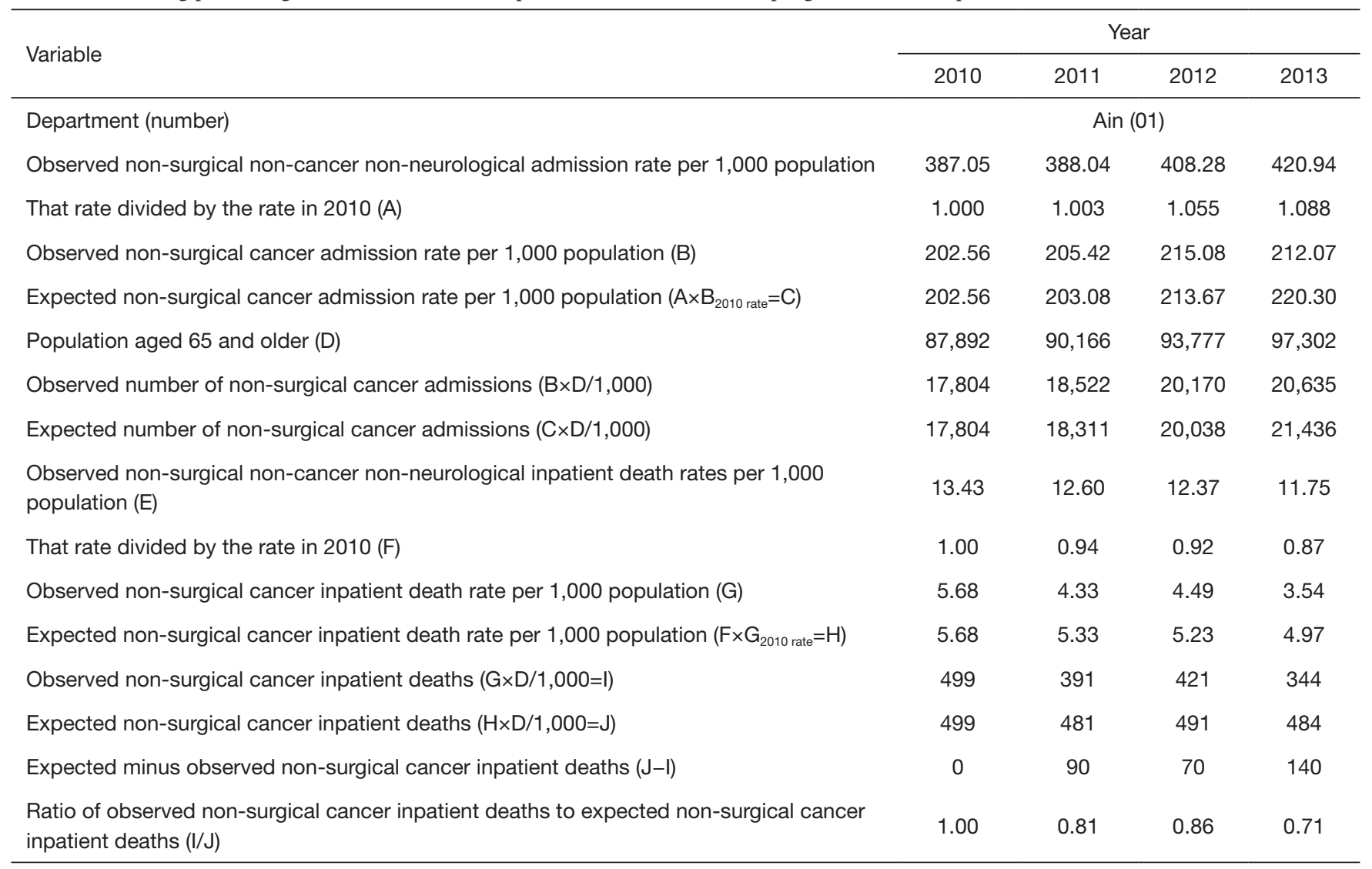

is demonstrated in Table 2.

The study and its use of anonymized data was approved by the French National Union of Regional Health Observatories (Fédération Nationale des Observatoires Régionaux de la Santé) and the French IRB (Commission Nationale Informatique et Libertés, National Committee for Data Files and Individual Liberties) (CNIL authorization number 1180745).

\section{Results}

Over the study period, our model predicted that, had nonsurgical cancer diagnosis admission rates tracked those of non-surgical non-cancer and non-neurological admission rates, patients admitted with a cancer diagnosis would have been expected to experience 207,512 inpatient deaths; however, 187,118 inpatient deaths were observed, which 
Table 3 National estimates of observed, expected, and expected minus observed numbers of inpatient deaths for non-surgical admissions with primary diagnoses of cancer or neurological diseases in 2010-2013

\begin{tabular}{|c|c|c|c|c|c|}
\hline Measures & \multicolumn{4}{|c|}{ Year } & Cumulative \\
\hline \multicolumn{6}{|l|}{ Admissions with cancer diagnoses } \\
\hline Expected inpatient deaths & 50,646 & 50,595 & 53,073 & 53,197 & 207,512 \\
\hline Observed inpatient deaths & 50,646 & 43,801 & 45,401 & 47,269 & 187,118 \\
\hline $\begin{array}{l}\text { Expected minus observed inpatient deaths as a percentage of expected } \\
\text { inpatient deaths }(\%)\end{array}$ & 0 & 13.4 & 15.2 & 11.2 & 13.0 \\
\hline \multicolumn{6}{|l|}{ Admissions with neurological disease diagnoses } \\
\hline Expected inpatient deaths & 3,711 & 3,697 & 3,880 & 3,888 & 15,176 \\
\hline
\end{tabular}

Percentage of inpatient deaths avoided, numbers may not add exactly due to rounding.
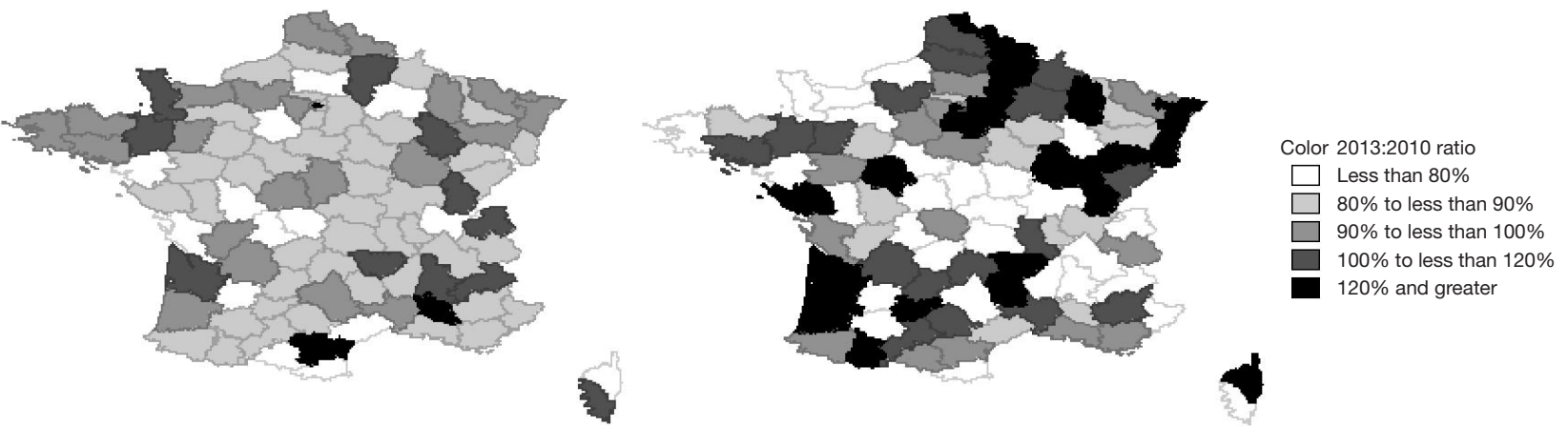

Figure 1 2013:2010 ratio of observed-to-expected rates of non-surgical admissions for cancer (A) or neurological disease (B) in which the patient died in the hospital. From lightest to darkest, the colors represent a 2013:2010 ratio of less than $80 \%$, $80 \%$ to less than $90 \%$, $90 \%$ to less than $100 \%, 100 \%$ to less than $120 \%$, and $120 \%$ or greater. A $2013: 2010$ ratio of $80 \%$ means that the ratio of actual to expected inpatient deaths decreased by $20 \%$ between 2010 and 2013; a 2013:2010 ratio of $120 \%$ means that the ratio of actual to expected inpatient deaths increased by $20 \%$ between 2010 and 2013 .

was 20,394 (13.0\%) fewer than expected (Table 3). The model also predicted that patients admitted with a primary neurological disease diagnosis would have been expected to experience 15,176 inpatient deaths; however, 14,663 inpatient deaths were observed, which was 513 (4.5\%) fewer than expected.

Observed-to-expected inpatient deaths fell more dramatically and more consistently for patients admitted with cancer diagnoses than for patients admitted with neurological diseases (Figure 1). Seemingly, observed-toexpected ratios fell the least (or even rose) in departments that were on the periphery of the French mainland when compared to departments in the French mainland's center. 


\section{Discussion}

\section{Principal findings}

In light of recent efforts to reduce inpatient deaths among patients with cancer and neurological disease diagnoses in France, we examined changes in the ratio of actual to expected inpatient deaths for such patients who were aged 65 and older and admitted for non-surgical reasons between 2010 and 2013. We found that observed inpatient deaths were below expected inpatient deaths for both types of admissions, considerably so for patients admitted with a cancer diagnosis.

These findings suggest that policies designed to emphasize community-based palliative care and reduce inpatient deaths among such patients have been effective. Indeed, those policies seem to have freed up substantial resources, potentially avoiding nearly 21,000 admissions in which the patient might have been expected to die over a 3 -year period.

Nonetheless, we found performance differences at the department level across France; while some departments demonstrated substantial reductions in the observed-toexpected number of inpatient deaths, other departments had substantial increases in that ratio. Reductions were more consistent across France when inpatient deaths among non-surgical admissions with primary cancer diagnoses. The second French National Cancer Plan, enacted between 2009 and 2013, that was intended to improve coordination between palliative care and cancer professionals' health networks and implemented a personal care plan for every cancer patient, could explain our findings that the greatest effects were among cancer patients (13).

\section{Strengths and weaknesses}

Our study has several limitations. First, like all studies that use administrative databases, our study assumes that coding is correct. To the extent data are miscoded, our findings are flawed. However, the general consistency of findings across years suggests that coding is consistent. Second, we did not have access to data that might explain the demand for healthcare services, such as underlying rates of year and department-specific cancer or neurological disease incidence or prevalence. Such data might help explain changes in admissions for the conditions we studied. Third, we did not have direct access about palliative care provided in the community. While we infer that community-based palliative care is the driver of changes in inpatient deaths, we cannot prove that.

However, the strengths of our study included our ability to study all relevant admissions in mainland France for 4 years, to correct for underling changes in admission practices during the time period examined, and to conduct small area variation analyses that can identify specific areas for improvement.

\section{Implications}

Our findings suggest that efforts to redeploy palliative care into the community have been effective. However, our findings also suggest that some departments may need help in achieving optimal performance levels. Policymakers might consider partnering teams from departments that demonstrated outstanding performance with teams from departments that appeared to have less effective results in order to accelerate change.

\section{Conclusions}

In France, efforts to reduce inpatient death rates among patients with cancer or neurological disease diagnoses appear to be effective. However, the effectiveness of those efforts varies geographically, suggesting that targeted efforts to improve lower performing departments may generate substantial performance improvements.

\section{Acknowledgements}

Dr. Weeks was funded by a Fulbright-Tocqueville grant through the Franco-American Commission for Educational Exchange and by the Institute of Advanced Studies at AixMarseille University.

\section{Footnote}

Conflicts of Interest: The authors have no conflicts of interest to declare.

Ethical Statement: The study and its use of anonymized data was approved by the French National Union of Regional Health Observatories (Fédération Nationale des Observatoires Régionaux de la Santé) and the French IRB (Commission Nationale Informatique et Libertés, National Committee for Data Files and Individual Liberties) (CNIL authorization number 1180745). 


\section{References}

1. Davies E, Higginson IJ. Better Palliative Care for Older People. Copenhagen, Denmark: World Health Organization; 2004 [cited 2016 June 2]. Available online: http://www.euro.who.int/_data/assets/pdf_ file/0009/98235/E82933.pdf

2. Broad JB, Gott M, Kim H, et al. Where do people die? An international comparison of the percentage of deaths occurring in hospital and residential aged care settings in 45 populations, using published and available statistics. Int J Public Health 2013;58:257-67.

3. Baumann A, Audibert G, Claudot F, et al. Ethics review: end of life legislation--the French model. Crit Care 2009;13:204.

4. Programme de développement des soins palliatifs.: Ministère des Affaires Sociales et de la Santé, Paris 2008 [updated le 13 Juin, 2008; cited 2016 June 2]. Available online: http://social-sante.gouv.fr/IMG/pdf/Programme_ de_developpement_des_soins_palliatifs_2008_2012.pdf

5. Observatoire National de la Fin de Vie [cited 2016 May 15]. Available online: http://www.onfv.org/

6. Circulaire $21 / 20$ du 04 Février 2003 relative à application de la circulaire DAR n5-2000 du 22 Mars 2000. Paris: L'Assurance Maladie des Salariés-Sécurité Sociale, Caisse Nationale; 2003 [cited 2016 June 2]. Available online: http://social-sante.gouv.fr/IMG/pdf/Circulaire_de_la_ CNAM_noCIR-21-2003_en_date_du_4_fevrier_2003_ relative_au_financement_du_dispositif_de_maintien_a_ domicile_dans_le_cadre_des_soins_palliatifs.pdf

7. Ministère des Affaires sociales et de la Santé. Manuel des groupes homogènes de malades. 11 ème version de

Cite this article as: Weeks WB, Ventelou B, Bendiane MK. Is the French palliative care policy effective everywhere? Geographic variation in changes in inpatient death rates among older patients in France, 2010-2013. Ann Palliat Med 2016;5(4):242-247. doi: 10.21037/apm.2016.08.05 la classifcation, 6ème revision (11g). Version 13.11g de la fonction groupage. Volume 1: Présentation et annexes générales. Section 1.2.2. [cited 2016 May 15]. Available online: http://www.atih.sante.fr/sites/default/files/public/ content/2708/volume_1.pdf 2015

8. Weeks WB, Jardin M, Dufour JC, et al. Geographic variation in admissions for knee replacement, hip replacement, and hip fracture in France: evidence of supplier-induced demand in for-profit and not-for-profit hospitals. Med Care 2014;52:909-17.

9. Weeks WB, Jardin M, Paraponaris A. Characteristics and patterns of elective admissions to for-profit and not-forprofit hospitals in France in 2009 and 2010. Soc Sci Med 2015;133:53-8.

10. Weeks WB, Paraponaris A, Ventelou B. Geographic variation in rates of common surgical procedures in France in 2008-2010, and comparison to the US and Britain. Health Policy 2014;118:215-21.

11. The Dartmouth Atlas of Health Care: Indirect Adjustment [cited 2016 March 8]. Available online: http://www. dartmouthatlas.org/downloads/methods/indirect_ adjustment.pdf

12. Estimmation de la population au 1er janvier par région, dépeartment (1975-2014), sexe et âge (quinquennal, classses d'âge). Paris, France: INSEE - Institut national de la statistique et des études économiques; 2015 [cited 2016 January 14]. Available online: http://www.insee.fr/fr/ themes/detail.asp?reg_id=99\&ref_id=estim-pop

13. Plan cancer, 2009-2013. Paris: Institut National du Cancer; 2013 [cited 2016 June 2]. Available online: http://social-sante.gouv.fr/IMG/pdf/Synthese_plan_ cancer_2009_2013.pdf 